\title{
Paramagnetic Contribution to the Magnetic Susceptibility of Bechgaard Salts.
}

\author{
A. L. TChougreefF and I. A. Misurkin \\ Karpov Institute of Physical Chemistry - 103064 Moscow K-64, ul. Obukha 10, Russia
}

(ricevuto il 20 Giugno 1991; revisionato il 28 Aprile 1992; approvato l'8 Maggio 1992)

\begin{abstract}
Summary. - The crystal of Bechgaard salt ((TMTSF $\left.)_{2} \mathrm{X}\right)$ is considered as a system of defect-bounded finite-length fragments of the TMTSF stacks. The paramagnetic contribution $\chi_{\text {spin }}$ to the susceptibility of the system arises due to the thermal population of the triplet excited states of the fragments and considerably increases with temperature in accordance with experiment. The unusual dependence of the pressure fractional derivative of $\chi_{\text {spin }}$ on temperature is explained as well. For the average fragment length flowing to infinity our expression for $\chi_{\text {spin }}$ transforms into the known Pauli formula and becomes temperature independent.
\end{abstract}

PACS 75.20 - Diamagnetism and paramagnetism.

PACS 73.20.Dx - Electron states in low-dimensional structures (including quantum wells, superlattices, layer stuctures, and intercalation compounds).

\section{1. - Introduction.}

The charge-transfer crystals similar to Bechgaard salts (TMTSF) 2 X (TMTSF is tetramethyltetraselenafulvalene, $\mathrm{X}$ is an inorganic anion like $\mathrm{PF}_{6}^{-}, \mathrm{AsF}_{6}^{-}$, or $\mathrm{SbF}_{6}^{-}$) are usually considered as «organic metals». Such an approach is usually justified by the character of the temperature dependence of electric conductivity. It drastically increases (by two orders of magnitude) while the temperature decreases from room temperature to about $20 \mathrm{~K}$. On the other hand, the magnetic susceptibility $\chi$ even qualitatively strongly differs from the Pauli susceptibility of normal metals. At temperatures about $T_{\mathrm{N}} \approx(10 \div 15) \mathrm{K}$ and at ambient pressure Bechgaard salts experience a transition into the antiferromagnetic state with a spin density wave (SDW). Below $T_{\mathrm{N}}$ (the Neel temperature) the total magnetic susceptibility decreases remaining finite at zero temperature as should be in the common antiferromagnet. Above $T_{\mathrm{N}}$ the susceptibility remains approximately constant up to about $50 \mathrm{~K}$ and then increases with temperature [1-4] in contrast with normal metals. The susceptibility of the so-called «organic metals» strongly depends also on pressure.

In general the behaviour of all organic metals and in particular of Bechgaard salts (BS) hardly fits into any existing theoretical model. At low temperatures and 
ambient pressure BS are antiferromagnets. All the body of data concerning the conductivity, ESR and NMR relaxation times, static magnetic moments etc.[1] strongly supports the idea of the SDW character of the low-temperature insulating state.

In the literature two viewpoints upon the discrepancy between the metallic type of conductivity and obviously the nonmetallic type of susceptibility are presented. Some authors consider high conductivity in terms of the simple-band theory with highly anisotropic energy bands. The values of the mean free path determined by low-temperature conductivity amount up to $10^{4} \AA$ thus implying the defect concentration in the BS crystal to be of the order of 0.1 mole\%. This value seems, however, to be unnatural in view of the way the material is obtained. It is synthesized by means of electrochemical precipitation from the solution at room temperature and therefore considerable defect concentration should arise. We assume it to be of the order of 1 mole\%. The high conductivity of BS at low temperatures in its turn can be attributed to superconductive fluctuations occurring above the superconductive critical temperature [5] rather than to the large mean free path.

The authors of [6-9] studied the changes in low-temperature conductivity, Hall effect, magnetoresistance, and in SDW transition temperature of BS induced by irradiation damage. These experiments have been interpreted in [6] by mean of the model with weakly interacting segments proposed in [10]. The model[10], however, does not go beyond the scope of the model with metallic segments proposed in $[11,12]$ for electronic properties of quasi-one-dimensional materials. According to $[6,10]$ the irradiated crystal of a quasi-one-dimensional material is an assembly of metallic segments bounded by defects. The interaction between the segments is weak. That idea seems to be very nice. At the same time it seems to be logical to consider any quasi-one-dimensional material as the assembly of segments without any irradiation. We think (in line with [11]) that some fraction of defects leading to fragmentation of the idealized infinite stacks arises in the course of preparation of the crystals. The data obtained in irradiation experiments [7-9] can be used to support this viewpoint by the following reasoning.

It is known that electronic states of the infinite one-dimensional stack are delocalized. Defects drastically change the electronic structure of the one-dimensional system. Even a small concentration of defects leads to the localization of its electronic states (for review see[13]) and, respectively, even a small irradiation damage has to result in sharp changes in transport and other electronic properties of quasi-one-dimensional materials. In contrast (as is seen from [7-9]) all the properties of quasi-one-dimensional materials vary smoothly with the increase of the irradiation dose from zero. No sharp transition from neat to irradiated crystals is observed. This suggests that irradiation merely provides additional defects to those which persisted in the crystal before and arose during the preparation. The zero irradiation dose in [7-9] does not correspond to zero but to some finite defect concentration ( $c=c_{0} \approx$ $\approx 1$ mole $\%$ ) which is inherent in neat materials. The zero defect concentration where the sharp transition is expected cannot be reached experimentally.

Another important notion concerning the models [6-12] is that the one-dimensional stack itself is not metallic. The separate stack (as well as the separate fragment of the stack) cannot be treated as a conducting metallic wire. More likely it should be considered as an array of sites with electrons occupying the sites. The Hamiltonian for that array should include the intersite hopping and on-site electron-electron 
repulsion. That model in the one-dimensional case straightforwardly leads to an insulating (rather than metallic) state for the stack even in the case when the repulsion parameter is small as compared with the parameter of the intersite hopping [14]. This state is referred to as the spin density wave (SDW) or the Mott insulating state of the one-dimensional Hubbard model (for review, see $[1,15]$ ).

All the above considerations suggest that the state of the separate fragment of the stack is the Mott insulating state rather than the metallic state in contrast with the suggestion of paper [6]. The explanation of the contradiction is the one-dimensionality of the stack. Even for $t_{\|} / \gamma \gg 1\left(t_{\|}\right.$is the intersite hopping and $\gamma$ is the on-site repulsion) the one-dimensional system remains insulating [14]. It should be noted, however, that in the framework of the model under consideration the intrinsic electronic structure of the fragment only slightly affects the conductivity of the crystal which is determined mainly by interfragment hopping [6,8]. On the other hand, the proposed approach makes it possible to explain the existence of the low-temperature ordered states (for more details, see[16]).

The model of metallic segments has been applied to the calculation of the magnetic susceptibility of quasi-one-dimensional materials [12]. It has been found that the susceptibility of the assembly of metallic segments is of the Pauli form with the effective density of states which takes into account the distribution of the fragments with respect to their lengths. Clearly such a result cannot account for the temperature behaviour of the experimentally observed susceptibility [1-4]. In the present paper the susceptibility is considered as the average susceptibility of fragments of different length. However, the fragments themselves are treated as the Mott insulating ones. The thermally populated triplet excited states of the fragments give a contribution to the temperature dependence of the susceptibility.

\section{2. - Theory.}

It is known (see ref.[1]) that the BS crystals consist of one-dimensional stacks formed by the (TMTSF) ${ }_{2}^{+}$dimers and by the chains of the $\mathrm{X}^{-}$anions. According to [5] the electronic structure of BS can be described if one assumes the concentration of electrons to be one electron per (TMTSF) ${ }_{2}^{+}$unit. So we assume that each fragment containing $N$ dimers (sites) can be described with use of the $N$-site Hubbard model with one electron per site (see $[1,15])$.

2*1. Triplet excitations of fragment. - The triplet excitation spectrum of the separate fragment in the Mott insulating (SDW) state has been derived in ref. [17]. For the cyclic $N$ site system the energies of the triplet excitations have the form

$$
\varepsilon_{n}=\frac{u}{N}|n|, \quad u=4 \pi t_{\|} \frac{I_{1}\left(2 \pi t_{\|} / \gamma\right)}{I_{0}\left(2 \pi t_{\|} / \gamma\right)}, \quad n= \pm 1, \pm 2, \ldots,
$$

where $t_{\| \mid}$is the intersite electron hopping parameter, $\gamma$ is the on-site electron-electron repulsion parameter, $I_{0}$ and $I_{1}$ are the modified Bessel functions. It has been shown in paper [18] that the triplet excitations in the $N$-site system with ends (i.e. in the linear fragment of $N$ sites) have the same form for large $N$.

22. The spin susceptibility of the fragment. - The spin susceptibility of a separate fragment is conditioned by the thermal population of its triplet states. According 
to [19] the susceptibility of the fragment of length $N$ is given by the formula

$$
\chi(N)=\frac{\mu_{\mathrm{B}}^{2} g_{\mathrm{e}}^{2}}{2 k T} \sum_{n=1}^{\infty} \operatorname{sech}^{2} \frac{n u}{2 N k T},
$$

where $\mu_{\mathrm{B}}$ is the Bohr magneton, $g_{\mathrm{e}}$ is the electronic $g$-factor, $k$ is the Boltzmann constant, $T$ is the temperature. In ref. [19] also two important asympthotics of eq. (2) have been found. If $u / 2 N k T \gg 1$ (short fragments and/or low temperature) the following estimate is valid:

$$
\chi<(N)=\frac{2 \mu_{\mathrm{B}}^{2} g_{\mathrm{e}}^{2}}{k T} \exp \left[-\frac{u}{N k T}\right] .
$$

The sense of this formula is quiet clear. It presents paramagnetic susceptibility due to a unique thermally populated triplet state with energy $u / N$.

If $u / 2 N k T \ll 1$ (long fragments and/or high temperature) the following estimate is valid:

$$
\chi_{>}(N)=\frac{\mu_{\mathrm{B}}^{2} g_{\mathrm{e}}^{2} N}{u} .
$$

Carefully analysing eq. (1) we can elucidate the details of the behaviour of $\chi>(N)$ close to the limit where the approximation equation (4) is valid. It reads

$$
\chi_{>}(N)=\mu_{\mathrm{B}}^{2} g_{\mathrm{e}}^{2}\left(\frac{N}{u}-\frac{1}{4 k T}\right)
$$

23. The spin contribution to the total susceptibility. - Now let us consider the contribution of the spin susceptibility to the mole susceptibility $\chi_{\text {spin }}$. As one can easily prove

$$
\chi_{\text {spin }}=\frac{N_{\mathrm{A}}}{\langle N\rangle} \int_{0}^{\infty} \chi(N) \rho(N) \mathrm{d} N,
$$

where $N_{\mathrm{A}}$ is the Avogadro number,

$$
\langle N\rangle=\int_{0}^{\infty} N_{\rho}(N) \mathrm{d} N
$$

is the average number of dimers in the fragment (i.e. the average number of sites or the average length), $\rho(N)$ is the partition function of fragments with respect to their lengths.

Both the long and the short fragments contribute to the mole spin susceptibility. Contributions from the areas where one of the asymptotical formulae is valid can be found straightforwardly. Questions arise when we intend to interpolate the values of $\chi(N)$ in the intermediate range of $N$ where both the conditions $u / 2 N k T \gg 1$ and $u / 2 N k T \ll 1$ are not satisfied.

To fill the intermediate $N$ region, we choose the characteristic fragment length $N^{*}=u / k T$ which is temperature dependent. Equations (3) and (5) are, respectively, valid if $N \ll N^{*}$ and $N \gg N^{*}$. Substituting the value $N^{*}$ into eqs. (3), (5), we obtain 
two estimates for the susceptibility of the fragment of the length $N^{*}$ :

$$
\chi_{<}\left(N^{*}\right)=\frac{2 \mu_{\mathrm{B}}^{2} g_{\mathrm{e}}^{2}}{e k T} \quad \text { and } \quad \chi>\left(N^{*}\right)=\frac{3 \mu_{\mathrm{B}}^{2} g_{\mathrm{e}}^{2}}{4 k T} .
$$

These values coincide with an accuracy of several per cent thus suggesting that the asymptotic formulae are good enough for our purposes even for the intermediate values of $N$. We can write

$$
\chi(N)= \begin{cases}\chi<(N), & \text { if } N<N^{*} \\ \chi>(N), & \text { if } N>N^{*}\end{cases}
$$

Inserting this expression into eq. (6) we obtain

$$
\chi_{\text {spin }}=\chi_{\text {long }}+\chi_{\text {short }} \text {, }
$$

where

$$
\chi_{\text {short }}=\frac{N_{\mathrm{A}}}{\langle N\rangle} \int_{0}^{N^{*}} \chi_{<}(N) \rho(N) \mathrm{d} N, \quad \chi_{\text {long }}=\frac{N_{\mathrm{A}}}{\langle N\rangle} \int_{N^{*}}^{\infty} \chi>(N) \rho(N) \mathrm{d} N .
$$

Taking the partition function in the form

$$
\rho(N)=1 /\langle N\rangle \exp [-N /\langle N\rangle],
$$

we estimate the first integral using the Laplace method (see also paper[20]):

$$
\chi_{\text {short }}=\frac{2 N_{\mathrm{A}} \mu_{\mathrm{B}}^{2} g_{\mathrm{e}}^{2}}{\langle N\rangle k T}\left(\frac{\pi^{2} u}{\langle N\rangle k T}\right)^{1 / 4} \exp \left[-2\left(\frac{u}{\langle N\rangle k T}\right)^{1 / 2}\right] .
$$

For the second one the integration can be carried out explicitly:

$$
\chi_{\text {long }}=\frac{N_{\mathrm{A}} \mu_{\mathrm{B}}^{2} g_{\mathrm{e}}^{2}}{\langle N\rangle u}\left(\langle N\rangle+\frac{u}{k T}+1\right) \exp \left[-\frac{u}{\langle N\rangle k T}\right] .
$$

For $\langle N\rangle \rightarrow \infty$ eq. (9) transforms into an expression independent of temperature $\left(\chi_{\text {short }}\right.$ vanishes in this limit):

$$
\chi_{\infty}=\frac{N_{\mathrm{A}} \mu_{\mathrm{B}}^{2} g_{\mathrm{e}}^{2}}{u} .
$$

It coincides with the well-known Pauli result

$$
\chi_{\text {Pauli }}=\frac{N_{\mathrm{A}} \mu_{\mathrm{B}}^{2} g_{\mathrm{e}}^{2}}{4} g\left(\varepsilon_{\mathrm{F}}\right),
$$

provided the effective density of states at the Fermi level $g\left(\varepsilon_{F}\right)=4 / u$. For noninteracting electrons $(\gamma=0)$ in the half-filled one-dimensional band the two formulae give the same result:

$$
\chi_{\text {band }}=\frac{N_{\mathrm{A}} \mu_{\mathrm{B}}^{2} g_{\mathrm{e}}^{2}}{4 \pi t_{\|}},
$$


because $u=4 \pi t_{\|}$for $\gamma=0$ (eq. (1)) and $g\left(\varepsilon_{\mathrm{F}}\right)=\left(\pi t_{\|} \sin k_{\mathrm{F}}\right)^{-1}, k_{\mathrm{F}}=\pi / 2$. The latter expression eq. (12) is usually used in order to describe experimental data on susceptibility of organic metals $[1,3,4]$. As we have shown this expression appears only in the limit $\langle N\rangle \rightarrow \infty$ and $\gamma \rightarrow 0$ of eq. (7) and thus can be merely a crude estimate of the susceptibility. From eq. (1) one can easily see that Coulomb interaction leads to an increase of the effective density of states and thus to an enhancement of the susceptibility over its value for noninteracting band electrons.

24. Parameters and calculations. - We used eqs. (7)-(9) to calculate the paramagnetic contribution to the total susceptibility of BS. The parameters were taken from paper[16] where a similar parameter set was used to describe the low-temperature electronic ordered states of BS (antiferromagnetic and superconductive states). According to [16] we set $t_{\|}=1500 \mathrm{~K}, \gamma=6000 \mathrm{~K}$, and $\langle N\rangle=40$.

24.1. Temperature dependence of the susceptibility. We calculated $\chi_{\text {spin }}$ for temperatures ranging from 100 to $290 \mathrm{~K}$ by eqs. (7)-(9). The Laplace method estimate, eq. (8), is valid when $\langle N\rangle<N^{*}$ only. It can be easily proven that this condition is satisfied for the parameters values chosen above. The found $\chi_{\text {spin }}$ for (TMTSF) $)_{2} \mathrm{X}$ is $1.52 \cdot 10^{-4}$ e.m.u. $/ \mathrm{mol}$ at $T=290 \mathrm{~K}$. This value perfectly agrees with the estimate given in review [1] for the susceptibility of the separate TMTSF stack, which equals $1.65 \cdot 10^{-4}$ e.m.u./mol. Analysis of the experimental on the spin susceptibility of BS is complicated by the large uncertainty in the core diamagnetic contribution $\chi_{\mathrm{D}}$. The estimate of $\chi_{\mathrm{D}}$ obtained by using the Pascal coefficients given in [2] is $-4.55 \cdot 10^{-4}$ e.m.u./mol. The authors of [2] consider it as an overestimate and argue that it is to be reduced by about $10^{-4}$ e.m.u./mol in absolute value. The values of the spin susceptibility of (TMTSF) $)_{2} \mathrm{X}$ at $290 \mathrm{~K}$ are obtained from the total susceptibility measured in $[3,4]$ by the Faraday balance method by using the diamagnetic susceptibility values which are close to that used in [2]. After reducing them by $1.0 \cdot 10^{-4}$ e.m.u./mol to obtain the true spin contribution, the values of the spin susceptibility [3,4] at $290 \mathrm{~K}$ fall in the range from $1.7 \cdot 10^{-4}$ to $2.3 \cdot 10^{-4}$ e.m.u./mol for different anions $\mathrm{X}^{-}$and agree with the results of ref.[2]. So our theoretical results are in reasonable agreement with the experimental data of ref. [2-4].

Contrarily, the above parameter set, used for calculation of the spin susceptibility in the framework of the model with noninteracting band electrons (eq. (12)), gives the value $0.8 \cdot 10^{-4}$ e.m.u. $/ \mathrm{mol}$. This estimate is twice as low as the value obtained from eqs. (7)-(9). Therefore the proposed model (eqs. (7)-(9)) describes the enhancement $[3,4]$ of the experimental value of susceptibility over the band value as well.

The calculated numerical values of the spin contribution to the susceptibility are of little importance themselves. An accurate qualitative behaviour of the susceptibility under pressure (see next section) and the correct form of its temperature dependence are much more important. The calculated spin contribution to the susceptibility smoothly increases with temperature (see fig. 1) as the experimental susceptibility does. At ambient pressure $\chi_{\text {spin }}$ increases by the factor 1.9 , while the temperature increases from $100 \mathrm{~K}$ to $290 \mathrm{~K}$ (the experiment [3] gives for BS the factor 1.6). We think that the temperature-independent difference between the calculated and observed values of the spin susceptibility is not very important. It 


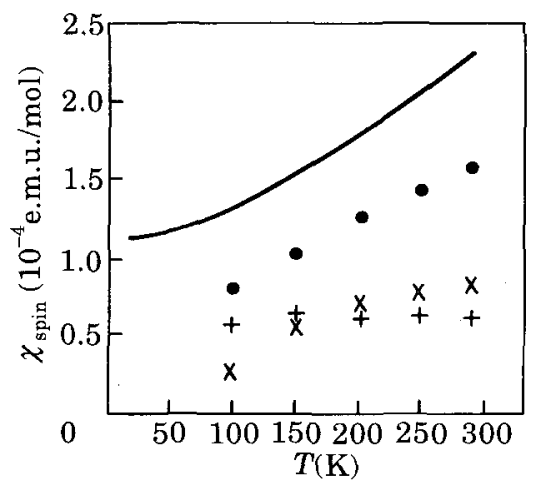

Fig. 1. - The temperature dependence of the spin susceptibility. The solid line is the spin susceptibility of (TMTSF) ${ }_{2} \mathrm{PF}_{6}$ measured in ref.[2] by the ESR intensity. Solid dots (•) represent the spin susceptibility $\left(\chi_{\text {spin }}\right)$ calculated as a sum of the contributions from short $(+)$ and long $(x)$ fragments.

may be attributed, for example, to the same uncertainties in the diamagnetic contribution.

24.2. Pressure dependence of susceptibility. The unique quantity in the proposed theory which depends on the crystal lattice parameters is the characteristic energy $u$ being in its turn the function (eq. (1)) of the electronic parameters $t_{\|}$and $\gamma$. The hopping parameter $t_{\|}$obviously increases with pressure due to decrease of the intermolecular separations. The pressure dependence of the on-site repulsion parameter $\gamma$ is not so clear. Though it should not be strong since $\gamma$ is a molecular property [4] there are some reasons $[5,16]$ to think that $\gamma$ decreases with pressure. Variations of both $t_{\|}$and $\gamma$ with pressure cause an increase of $u$ (eq. (1)).

From the analysis of eqs. (7)-(9) one can easily conclude that $\chi_{\text {spin }}$ decreases while $u$ increases. Carefully inspecting eqs. (7)-(9), one can note that the temperature increase damps the effect of the $u$ variation on the susceptibility.

We calculated $\chi_{\text {spin }}$ as a function of pressure assuming the pressure dependence of $t_{\|}$to be linear with slope $t_{\|}^{\prime}$ of $20 \mathrm{~K} / \mathrm{kbar}$. The latter value is only slightly higher than that of $1 \% / \mathrm{kbar}$ proposed in [4]. Our calculations show that $\chi_{\text {spin }}$ indeed decreases with increasing pressure. The $\left|\mathrm{d} \ln \chi_{\text {spin }} / \mathrm{d} P\right|$ varies from $2.6 \% / \mathrm{kbar}$ at $290 \mathrm{~K}$ to $4 \% / \mathrm{kbar}$ at $100 \mathrm{~K}$. The experiments performed on $(\mathrm{TMTSF})_{2} \mathrm{PF}_{6}$ in $[3,4]$ give $2.8 \% / \mathrm{kbar}$ and $4.8 \% / \mathrm{kbar}$, respectively.

\section{3. - Discussion.}

In the present paper we refined the approach to the explanation of the temperature and pressure dependence of the Bechgaard salts (BS) paramagnetic susceptibility previously developed in papers [6-12]. Two ideas are crucial for our approach. The first is the idea of the structure defects in one-dimensional stacks of the donor molecules. The second is that of the Mott insulating state of the defect-bounded fragments. The two ideas contradict all the data on BS if the latter are understood in the framework of the simple band theory. We however have noted in the introduction that the interpretation of the experiments carried out on BS in terms of the band theory is not frequently unequivocal. For instance the bandwidth 
measured in the experiments on reflectance will be the same in the framework of the model with fragments because the zero reflectance energy corresponds to the transition between the lowest-energy occupied state and the highest-energy empty state, which all the same is $4 t_{\|}$for both the infinite stack and the finite fragment. Below that energy the reflectance spectrum is determined by transitions between the occupied and empty one-electron states within fragments. The fragments are long enough and their lengths are random. For these two reasons the one-electron states of the fragments are lying close in energy, thus giving wide structureless band in the reflectance spectra [1].

Some experimental data do not fit into the band theory at all. The properties of the ordered phases in BS at low temperature (see also [16] or the susceptibility considered in the present paper can be understood only in terms of the finite fragments (for review see[1]). And this is not surprising in view of recent findings due to Anderson[21]. He has shown that the quasi-one-dimensional Hubbard model has the property of «confinement» which means that the interstack hopping $t_{\perp}$ when it is weak as compared to the electron-electron repulsion $\gamma$ does not result in coherent motion of electrons in the transverse direction. According to [21] the two-dimensional $t_{\perp}-\gamma$ phase diagram of the quasi-one-dimensional Hubbard model is divided into two areas. The first area is that with $t_{\perp}>\gamma$. The second area corresponds to $t_{\perp}<\gamma$ and here the «confined» regime occurs. The electron parameters $\left(t_{\perp}\right.$ and $\left.\gamma\right)$ seem to fall into the confinement area on the phase diagram for the majority of «organic metals». For that reason Anderson [21] suggested to re-examine the data on the transport properties (conductivity, thermopower) in terms of the confined states. Clearly the new treatment will result in a description different from the traditional band interpretation of the transport properties[1], since the band theory obviously describes the opposite limit.

Careful analysis reveals that an additional variable may be important for the description of the real «organic metals", namely, the defect concentration $c$ (or the average fragment length $\langle N\rangle$ ). It does not enter either the band theory or the perturbative approach of Anderson at all. The effect of the nonzero defect concentration upon the electronic states of the stack basically coincides with that of electron repulsion; the electronic states in the stack with either nonzero repulsion or nonzero defect concentration becomes localized (for review see[13]). For that reason we think that the Anderson's analysis of the case of interacting electrons picks up to some extent the case of finite fragments as well. So we believe that the reinterpretation proposed by Anderson will lead to a description of the transport properties of "organic metals" in terms of local states, which also appear in the case of finite fragments. Furthermore, there are some indications that the interfragment hopping may give the thermal dependence of conductivity which is normally attributed to metals. For example, Sumi [22] has shown, that in some cases the diffusive mobility diverges at low temperature as $T^{-2}$ which may be well misinterpreted as metallic behaviour.

One of the attractive features of the models with finite fragments is their capability to treat neat and irradiated crystals in a uniform manner. The parameters involved in the description of the magnetic susceptibility in the present paper are close to those used in [16] to describe low-temperature antiferromagnetic and superconductive states.

The general picture of the magnetic-susceptibility temperature dependence in Bechgaard salts based upon the present paper and paper [16] looks as follows. Below 
the antiferromagnetic transition threshold $T_{\mathrm{N}}$ the total susceptibility has finite values down to zero temperature. Above $T_{\mathrm{N}}$ the susceptibility concerned with the SDW phase ordering decreases according to the Curie-Weiss law. Spin-(1/2) defects also give some contribution to the total susceptibility. However, the experimental data on the Curie tail are not sufficient[4] and we do not analyse them. At higher temperatures when the fragment SDW phases are disordered the thermal population of the triplet states of separate fragments becomes significant. Their contribution rapidly increasing with temperature is responsible for the observed temperature and pressure dependence of the susceptibility.

The most important difference between the present approach and that proposed in $[6,12]$ is the description of the electronic state of the separate fragment. The idea of the metallic fragments invoked in $[6,12]$ fails to explain both the low-temperature antiferromagnetic order and the temperature dependence of the magnetic susceptibility. Our approach uses the insulating SDW states to describe the electronic structure of the separate fragment. This approach is consistent with the well-known dielectrization of the metallic state in one dimension [15,23].

Previously theoretical models of two types both based upon the band picture have been considered [4] to explain the unusual magnetic properties of BS. The models of the first type attribute the observed enhancement of the susceptibility above the Pauli band value to the Coulomb interactions. The Coulomb interactions in different approximations indeed give rise to some enhancement of the susceptibility, but its pressure dependence remains unexplained. In our model this difficulty is naturally avoided and the pressure dependence of susceptibility is reproduced with reasonable values of the parameters.

The models of the second type ascribe the enhancement and the temperature dependence of the susceptibility to polaronic effects. However, the conditions for the polaron model validity are not satisfied in the case of Bechgaard salts [4] and therefore polaronic effects seem to be insufficient to explain the experiment.

The theory proposed in the present paper predicts strong dependence of the susceptibility on the average fragment length $\langle N\rangle$. As long as we know, investigations of the susceptibility dependence on the defect concentration have never been carried out. Irradiation induces magnetic defects and they will give contribution of the Curie type to the susceptibility masking the pure effect of fragment length decrease. Contrarily alloying introduces nonmagnetic defects which will not interfere with the susceptibility additionally. Therefore solid solutions with the sulphur analogs of the TMTSF molecules may be convenient objects for such investigations.

\section{4. - Conclusion.}

In the present paper we suceeded in describing the experimental data on the paramagnetic contribution to the magnetic susceptibility of Bechgaard salts in a wide range of temperature and pressure, assuming that length;

a) structure defects break the molecular stacks into fragments of finite

b) finite-length fragments of the stacks are in the Mott insulating (SDW) state; 
c) the spin susceptibility of each fragment is determined by the thermal population of the fragment triplet states.

$$
* * *
$$

The authors are grateful to the referee for the deep comments and to Prof. F. Bassani for encouragement and support.

\section{REFERENCES}

[1] D. Jerome and H. J. Schulz: Adv. Phys., 31, 299 (1982).

[2] J. C. Scott, H. J. Pedersen and K. Bechgandd: Phys. Rev. Lett., 45, 2125 (1980).

[3] L. Forro, J. R. Cooper, B. Rothaemel, J. S. Schilling, M. Weger, D. Schweitzer, H. J. Keller and K. BechgaARD: Synth. Metals, 19, 339 (1987).

[4] L. Forro, J. R. Cooper, B. Rothaemel, J. S. Schilling, M. Weger and K. Beckgaard: Solid State Commun., 60, 11 (1986).

[5] D. Jerome: Chem. Scripta, 17, 13 (1981).

[6] L. Zuppiroli: Ber. Bunsenges. Phys. Chem., 88, 304 (1984).

[7] L. Forro: Mol. Cryst. Liq. Cryst., 85, 315 (1982).

[8] L. Zuppiroli, S. Bouffard, K. BechGaard, B. Hilti and C. W. Mayer: Phys. Rev. B, 22, 6035 (1980).

[9] B. Korin-Hamzic, L. Forro, J. R. Cooper and K. Bechgando: Phys. Rev. B, 38, 11177 (1988).

[10] L. ZuPPIROLI: Radiat. Eff., 62, 53 (1982).

[11] D. Kuze and H. R. Zeller: Phys. Rev. Lett., 27, 1060 (1971).

[12] M. J. Rice and J. Bernasconi: J. Phys. F, 3, 55 (1973).

[13] Yu. A. Firsov, V. N. Prigodin and C. Seidel: Phys. Rep., 126, 245 (1985).

[14] E, H. LIEB and F, Y. Wu: Phys. Rev. Lett., 20, 1445 (1968).

[15] N. F. MotT: Metal-Insulator Transitions (Taylor \& Francis, 1974).

[16] A. L. TchougreefF and I. A. Misurkin: Fiz. Tverd. Tela, 30, 1043 (1988) (in Russian); Sov. Phys. Solid State, 30, 605 (1988) (in English).

[17] A. A. Ovchinnikov: Ž. $\dot{E} k s p$. Teor. Fiz., 57, 2137 (1969) (in Russian); Sov. Phys. JETP, 30, 1160 (1970) (in English).

[18] I. A. Misurkin, G. A. Vinogradov and A. A. Ovchinnikov: Int. J. Quantum. Chem., 9, 605 (1975).

[19] A. A. Berlin, G. A. Vinogradov and A. A. Ovchinnikov: Int. J. Quantum. Chem., 6, 263 (1972).

[20] G. A. Vinogradov, I. A. Misurkin and A. A. Ovchinnikov: Teor. Exp. Khim., 12, 723 (1976) (in Russian).

[21] P. W. Anderson: Phys. Rev. Lett., 67, 3844 (1991).

[22] H. Sumi: J. Chem. Phys., 70, 3775 (1979).

[23] R. PeIERLS: Quantum Theory of Solids (Clarendon Press, 1955). 